\title{
RNA-Seq based phylogeny recapitulates previous phylogeny of the genus Flaveria (Asteraceae) with some modifications
}

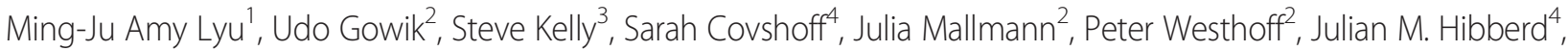 \\ Matt Stata ${ }^{5}$, Rowan F. Sage ${ }^{5}$, Haorong Lu' ${ }^{6}$ Xiaofeng Wei ${ }^{6}$, Gane Ka-Shu Wong ${ }^{6,7,8}$ and Xin-Guang Zhu ${ }^{1 *}$
}

\begin{abstract}
Background: The genus Flaveria has been extensively used as a model to study the evolution of $C_{4}$ photosynthesis as it contains $C_{3}$ and $C_{4}$ species as well as a number of species that exhibit intermediate types of photosynthesis. The current phylogenetic tree of the genus Flaveria contains 21 of the 23 known Flaveria species and has been previously constructed using a combination of morphological data and three non-coding DNA sequences (nuclear encoded ETS, ITS and chloroplast encoded trnL-F).

Results: Here we developed a new strategy to update the phylogenetic tree of 16 Flaveria species based on RNA-Seq data. The updated phylogeny is largely congruent with the previously published tree but with some modifications. We propose that the data collection method provided in this study can be used as a generic method for phylogenetic tree reconstruction if the target species has no genomic information. We also showed that a "F. pringlei" genotype recently used in a number of labs may be a hybrid between $F$. pringlei $\left(C_{3}\right)$ and $F$. angustifolia $\left(C_{3}-C_{4}\right)$.

Conclusions: We propose that the new strategy of obtaining phylogenetic sequences outlined in this study can be used to construct robust trees in a larger number of taxa. The updated Flaveria phylogenetic tree also supports a hypothesis of stepwise and parallel evolution of $C_{4}$ photosynthesis in the Flavaria clade.
\end{abstract}

Keywords: Flaveria, RNA-Seq, Phylogenetic tree, $C_{4}$ photosynthesis

\section{Background}

$\mathrm{C}_{4}$ photosynthesis evolved repeatedly from $\mathrm{C}_{3}$ photosynthesis in at least 66 different lineages of angiosperms [1,2]. Many of these evolutionary transitions are coincident with the decline of atmospheric $\mathrm{CO}_{2}$ concentration in the Oligocene $[1,3,4]$. Because of the $\mathrm{C}_{4}$ photosynthetic pathway, $\mathrm{C}_{4}$ plants are able to concentrate $\mathrm{CO}_{2}$ into the bundle sheath cells (BSC) where RuBisCO (ribulose 1,5bisphosphate carboxylase/oxygenase) is localized [5]. This substantially reduces the inhibitory process of photorespiration and thus increases light, water and nitrogen use efficiencies in $\mathrm{C}_{4}$ relative to $\mathrm{C}_{3}$ plants [6,7]. Since its discovery in the 1960's, there has been much interest in understanding how $\mathrm{C}_{4}$ plants evolved $[8,9]$. In addition to

\footnotetext{
* Correspondence: xinguang.zhu@gmail.com

${ }^{1}$ CAS-MPG Partner Institute and Key Laboratory for Computational Biology,

Shanghai Institutes for Biological Sciences, Shanghai, China

Full list of author information is available at the end of the article
}

being scientifically intriguing, a thorough understanding of the evolutionary process of $\mathrm{C}_{4}$ photosynthesis will also help guide efforts to engineer $C_{4}$ traits into $C_{3}$ plants and boost crop yield and resource use efficiencies $[1,10,11]$. In recent years, the incorporation of phylogenetic methods into $\mathrm{C}_{4}$ studies has greatly aided the evaluation of evolutionary hypothesis concerning when, where and how the $C_{4}$ pathway evolved $[1,12]$. Genera that include both $\mathrm{C}_{3}$ species and $\mathrm{C}_{4}$ species, as well as species with intermediate phenotypes between $\mathrm{C}_{3}$ and $\mathrm{C}_{4}$, such as Flaveria [13] and Heliotropium [14], have been of particular interest for the study of the evolution of $\mathrm{C}_{4}$ photosynthesis. Intermediate species have features of both $C_{3}$ and $C_{4}$ photosynthetic pathways [13] and often differ from $C_{3}$ species by possessing characteristics of $\mathrm{C}_{4}$ plants such as enhanced $C_{4}$ enzyme expression and activity $[15,16]$. A concern has been whether the intermediate species branch in sister positions to $\mathrm{C}_{3}$ and $\mathrm{C}_{4}$ clades that would support 
a conclusion of shared ancestry and thus that the intermediate reflects an evolutionary transitional state [11, 17], however, resolving the phylogenetic positions of intermediates often requires a data-rich phylogeny [18].

Because it has multiple $\mathrm{C}_{3}$ and $\mathrm{C}_{4}$ species, more known $\mathrm{C}_{3}-\mathrm{C}_{4}$ intermediate species than any other genera, and is relatively young (past 5 to 10 million years), the genus Flaveria has become the main model system for the study of $\mathrm{C}_{4}$ evolution in the eudicots $[10,19]$. For over 30 years, researches with Flaveria species have examined the structural, physiological, biochemical and molecular variation in their leaves and genomes [12, 15, 20-23]. Collectively, these studies led to the proposal that $\mathrm{C}_{4}$ characters are acquired in a step-wise manner during the evolution of $\mathrm{C}_{4}$ photosynthesis, and recently, the rise of $\mathrm{C}_{4}$ character states in Flaveria were proposed to follow a progressive, Mount Fuji adaptive landscape model [17]. In support of this step-wise transition, a comparative study of the localization of P-protein of glycine decarboxylase (GLDP, a key enzyme in the photorespiratory pathway) into different photosynthetic types of Flaveria showed that the restriction of GLDP to BSC occurs gradually in going from pure $C_{3}$ to intermediate species and then to $C_{4}$ species [24].

An important development in the understanding of $\mathrm{C}_{4}$ evolution in Flaveria has been the publication of a wellresolved phylogenetic tree of the genus and sister taxa $[18,25,26]$. The first phylogeny of Flaveria was published using morphological markers by Powell [25]. This was followed by a single gene tree by Kopriva et al., based on the H-protein of glycine decarboxylase [26]. While both trees were limited by relatively small data sets on which to infer relationships, they did indicate two major clades are present in Flaveria, with each containing $\mathrm{C}_{3}-\mathrm{C}_{4}$ intermediate species and $\mathrm{C}_{4}$ or $\mathrm{C}_{4}$-like species in distal positions of each clade $[25,26]$. This suggests the possibility of two independent origins of the $\mathrm{C}_{4}$ pathway in Flaveria.

The most widely accepted phylogenetic tree of Flaveria was constructed using three non-coding DNA sequences (which comprised the nuclear ribosome internal transcribed region, external transcribed region, and the chloroplast encoded trnL-F spacer region) combined with a number of morphological features, such as life history, leaf surface properties and capitulescence and so on [18]. The inclusion of the morphological characters resulted in a composite tree that is widely used to infer Flaveria relationships in order to test hypotheses of evolutionary origin using physiological, biochemical or molecular data sets $[18,25]$. However phylogenies based on non-coding regions and/or loci under selection can also potentially provide different interpretations due to varying selection pressures, demography, and selection sweeps [27, 28]. Moreover, phylogenetic trees inferred from different non- coding sequences have been shown to be incongruent with each other in numerous taxa such as the Gramineae [27]. It has also been shown that phylogenetic trees inferred from morphological data often conflict with trees inferred from molecular sequence data [18, 27, 29]. Considering the increasing interest in precisely mapping out the evolutionary steps for $\mathrm{C}_{4}$ photosynthesis and the importance of the Flaveria genus in studying $\mathrm{C}_{4}$ evolution, it is pertinent to re-evaluate the phylogeny of Flaveria using the information-rich data based on molecular sequences that has recently become available [30].

With the advent of low-cost sequencing technologies, there has been a rapid accumulation of molecular sequence data for non-model species, such as Flaveria. Those algorithms that utilize such sequence data to construct phylogenetic trees have been recently developed [31-33]. For example, using data matrix constructed based on assembled contigs from RNA-Seq reads, a robust phylogeny of 10 mosquito species was constructed [33]. The phylogeny of 16 lice (Insecta: Psocodea) was reconstructed by combing EST data and genomic DNA-Seq data [34], and the phylogeny of 21 species from class Actinopterygii were generated using different sources of data, including public genome sequences, EST, mRNA, transcriptome as well as cDNA and Unigenes [35].

In this work, we present a new method to use RNASeq data to generate phylogenetic trees, and then use it to reconstruct the phylogeny of Flaveria. First, we extracted phylogentically informative sites from RNA-Seq data by directly mapping sequence reads to coding sequences (CDS) of a fully sequenced reference species (in this case $A$. thaliana). Using this data set, we generated a phylogenetic tree of 16 Flaveria species, including representatives of $\mathrm{C}_{3}, \mathrm{C}_{3}-\mathrm{C}_{4}$ intermediate, $\mathrm{C}_{4}$-like and $\mathrm{C}_{4}$ species. The resulting tree is largely consistent with the most widely referenced Flaveria phylogeny [18] but with slight modifications. Using our mapping strategy we provide evidence that a " $F$. pringlei" accession used in this and many prior studies is a hybrid derived from a pure parent of $F$. pringlei $\left(\mathrm{C}_{3}\right)$ and a $F$. angustifolia $\left(\mathrm{C}_{3}-\mathrm{C}_{4}\right)$ parent.

\section{Results}

\section{Overview of RNA-Seq samples sequenced through Illumina platform}

To construct a phylogenetic tree of the genus Flaveria, we obtained Illumina RNA-Seq data from 17 Flaveria species $\left(3 \mathrm{C}_{3}, 4 \mathrm{C}_{4}, 7 \mathrm{C}_{3}-\mathrm{C}_{4}\right.$ and $3 \mathrm{C}_{4}$-like intermediate species) and 3 out-group species from the Asteraceae (Table 1). In total there were 37 RNA-Seq samples including 34 Flaveria samples and 3 samples of out-group species. The 37 RNA-Seq samples were obtained from two sources. Nineteen samples were obtained from the One Thousand Plants (1KP) Consortium (http://www.onekp.com/) which 
Table 1 RNA-Seq data and cross mapping result

\begin{tabular}{|c|c|c|c|c|c|c|c|}
\hline Sample & PS type & \# read & Average length & \# mapping read & $\%$ mapping read & \# target CDS & $\%$ mapping target \\
\hline \multicolumn{8}{|c|}{ Pair-end RNA-seq of from Illumina, read length: 75-90 bp (from 1KP) } \\
\hline F. cronquistii-j & a & $16,809,686.00$ & 90 & $7,902,191.00$ & $47.01 \%$ & $25,692.00$ & $72.60 \%$ \\
\hline F. cronquistii-m & a & $15,622,832.00$ & 90 & $8,114,254.00$ & $51.94 \%$ & $25,337.00$ & $71.60 \%$ \\
\hline F. pringlei-j & a & $20,064,474.00$ & 90 & $9,372,051.00$ & $46.71 \%$ & $26,209.00$ & $74.07 \%$ \\
\hline F. pringlei-m & a & $16,219,108.00$ & 90 & $9,150,289.00$ & $56.42 \%$ & $25,308.00$ & $71.52 \%$ \\
\hline F. angustifolia-j & b & $16,668,010.00$ & 90 & $7,983,526.00$ & $47.90 \%$ & $25,599.00$ & $72.34 \%$ \\
\hline F. angustifolia-m & $b$ & $18,085,350.00$ & 90 & $9,059,885.00$ & $50.10 \%$ & $24,877.00$ & $70.30 \%$ \\
\hline F. pubesens-j & $b$ & $18,897,990.00$ & 90 & $11,118,929.00$ & $58.84 \%$ & $25,933.00$ & $73.29 \%$ \\
\hline F. pubesens-m & $b$ & $20,703,102.00$ & 90 & $8,502,290.00$ & $41.07 \%$ & $25,438.00$ & $71.89 \%$ \\
\hline F. sonorensis-j & $b$ & $22,424,194.00$ & 90 & $11,170,838.00$ & $49.82 \%$ & $25,959.00$ & $73.36 \%$ \\
\hline F. palmeri-j & c & $19,329,884.00$ & 90 & $10,666,300.00$ & $55.18 \%$ & $25,669.00$ & $72.54 \%$ \\
\hline F. vaginata-j & c & $18,876,338.00$ & 90 & $8,830,209.00$ & $46.78 \%$ & $25,814.00$ & $72.95 \%$ \\
\hline F. bidentis-j & $d$ & $25,424,874.00$ & 90 & $13,020,754.00$ & $51.21 \%$ & $26,103.00$ & $73.77 \%$ \\
\hline F. bidentis-m & $d$ & $23,089,000.00$ & 90 & $12,163,057.00$ & $52.68 \%$ & $25,582.00$ & $72.29 \%$ \\
\hline F. kochiana-m & $d$ & $19,220,058.00$ & 90 & $10,881,823.00$ & $56.62 \%$ & $25,359.00$ & $71.66 \%$ \\
\hline F. trinervia-j & $d$ & $23,726,482.00$ & 90 & $11,295,399.00$ & $47.61 \%$ & $26,442.00$ & $74.72 \%$ \\
\hline F. trinervia-m & $d$ & $27,345,748.00$ & 90 & $13,030,756.00$ & $47.65 \%$ & $25,665.00$ & $72.53 \%$ \\
\hline H. autumnale & a & $25,213,280.00$ & 90 & $7,916,909.00$ & $31.40 \%$ & $26,039.00$ & $73.59 \%$ \\
\hline Ta. parthenium & a & $19,828,848.00$ & 75 & $5,069,369.00$ & $25.57 \%$ & $26,041.00$ & $73.59 \%$ \\
\hline Tr. duius & a & $23,106,402.00$ & 90 & $9,485,525.00$ & $41.05 \%$ & $26,235.00$ & $74.14 \%$ \\
\hline Average & & $20,560,824.20$ & & & $47.67 \%$ & & $73.78 \%$ \\
\hline \multicolumn{8}{|c|}{ Single-end RNA-seq from Illumina, read length: $100 \mathrm{bp}$ from (HHU). } \\
\hline F. pringlei $i^{\#}$ & a & $38,529,805.00$ & 90.1 & $20,920,082.00$ & $54.30 \%$ & $28,605.00$ & $80.84 \%$ \\
\hline F. robusta & a & $33,113,842.00$ & 90.1 & $9,625,516.00$ & $29.07 \%$ & $29,033.00$ & $82.05 \%$ \\
\hline F. angustifolia ${ }^{\#}$ & b & $31,408,476.00$ & 85.1 & $14,328,304.00$ & $45.62 \%$ & $28,533.00$ & $80.63 \%$ \\
\hline F. anomala & b & $31,056,596.00$ & 91.1 & $15,457,407.00$ & $49.77 \%$ & $26,676.00$ & $75.39 \%$ \\
\hline F. chloraefolia & $b$ & $39,911,614.00$ & 89.9 & $18,468,621.00$ & $46.27 \%$ & $28,375.00$ & $80.19 \%$ \\
\hline F. floridana & $b$ & $38,236,849.00$ & 84.9 & $18,685,391.00$ & $48.87 \%$ & $28,465.00$ & $80.44 \%$ \\
\hline F. pubescens ${ }^{\#}$ & $b$ & $29,940,352.00$ & 91.4 & $15,965,038.00$ & $53.32 \%$ & $28,957.00$ & $81.83 \%$ \\
\hline F. ramosissima ${ }^{\#}$ & $b$ & $35,283,647.00$ & 90.4 & $20,060,016.00$ & $56.85 \%$ & $29,010.00$ & $81.98 \%$ \\
\hline F. brownii & c & $43,802,834.00$ & 91.6 & $20,986,495.00$ & $47.91 \%$ & $28,180.00$ & $79.64 \%$ \\
\hline F. palmeri ${ }^{\#}$ & c & $27,804,586.00$ & 84 & $12,421,541.00$ & $44.67 \%$ & $28,926.00$ & $81.74 \%$ \\
\hline F. vaginata ${ }^{\#}$ & c & $35,000,281.00$ & 84 & $16,077,619.00$ & $45.94 \%$ & $28,772.00$ & $81.31 \%$ \\
\hline F. australasica & $d$ & $25,312,995.00$ & 84.1 & $10,357,274.00$ & $40.92 \%$ & $27,387.00$ & $77.40 \%$ \\
\hline F. bidentis ${ }^{\#}$ & $d$ & $34,333,242.00$ & 90.9 & $16,600,362.00$ & $48.35 \%$ & $27,731.00$ & $78.37 \%$ \\
\hline F. trinervia ${ }^{\#}$ & $d$ & $33,540,674.00$ & 91.2 & $19,511,743.00$ & $58.17 \%$ & $29,059.00$ & $82.12 \%$ \\
\hline Average & & $34,091,128.00$ & & & $47.86 \%$ & & 0.815 \\
\hline F. bidentis"-root & & $34,491,406.00$ & 91.8 & $16,020,332.00$ & $46.45 \%$ & $28,180.00$ & $79.68 \%$ \\
\hline F. bidentis"-shoot & & $36,588,034.00$ & 91.3 & $17,261,488.00$ & $47.18 \%$ & $28,465.00$ & $80.48 \%$ \\
\hline F. robusta"-root & & $38,514,685.00$ & 91.8 & $17,220,911.00$ & $44.71 \%$ & $28,772.00$ & $81.35 \%$ \\
\hline F. robusta -shoot $^{-}$ & & $23,089,000.00$ & 90 & $12,163,057.00$ & $52.68 \%$ & $25,582.00$ & $72.33 \%$ \\
\hline Average & & $20,440,345.00$ & & & $47.64 \%$ & & $78.46 \%$ \\
\hline
\end{tabular}

Note: Abbreviations: F: Flaveria, H: Helenium, Ta: Tanacetum, Tr: Tragopogon, -j/m: juvenile/mature leaf sample from 1KP, "\#: leaf sample from HHU. PS. (photosynthetic) type: a: $C_{3}$, b: $C_{3}-C_{4}$, c: $C_{4}$-like, d: $C_{4}$ 
were grown in the University of Toronto and 18 samples were obtained from Heinrich-Heine University (HHU) (Table 1). On average, each Flaveria RNA-Seq sample from the $1 \mathrm{KP}$ Consortium and HHU provided around 20.0 million (from 15.6 million to 27.3 million) and 27.3 million (from 23.1 million to 43.8 million) raw reads respectively (Table 1). In summary, the 34 RNA-Seq datasets of 17 Flaveria species covered plants grown in greenhouses at HHU (18 datasets) and the University of Toronto (16 datasets), and included six species with both samples from juvenile leaves and mature leaves, besides, the datasets were from two different sequencing protocols, i.e. pair-end reads (University of Toronto) and single-end reads (HHU) using Illumina sequencing (Methods).

\section{Mapping reads to minimal coding sequence set of Arabidopsis thaliana}

The estimated divergence time between Flaveria and A. thaliana is $\sim 120$ million years (mys) [36]. The long divergence time and hence different evolutionary histories may result in gene family expansion through duplications and thus influence correct reads mapping. In order to reduce the potential artifacts from reads crossmapping caused by paralogs, a minimal reference coding sequences ( $m-C D S)$ of $A$. thaliana was used as mapping template (see Methods). The $m$-CDS contained 26,152 coding sequences (CDS) that was constructed by removing redundant paralogs. During cross-species mapping of RNA-Seq reads to $m$-CDS, we mapped reads in protein-space using BLAT and only retained mapped reads with an estimated false positive discovery rate (FDR) below $1 \%(q=0.00745$, see Methods). All samples have similar percentages of reads mapping to the $m$-CDS: $40 \%-45 \%$ of reads from the $1 \mathrm{KP}$ Consortium samples can be mapped to $m$-CDS reference, and $43 \% \sim 56 \%$ of reads from HHU samples can be mapped to the reference (Table 1). Thus, around $50 \%$ of the total number of reads from all samples was used in this study. To further estimate the accuracy of cross species mapping, we used the information from paired reads to determine the percentage of reads that mapped in concordant pairs to the same gene. On average, $68 \%$ of all mapped reads mapped in pairs, of which $99 \%$ mapped concordantly to the same reference, suggesting a high reliability of cross species mapping.

\section{Cross-species CDS sequence extrapolation}

Having selected the high quality mapped reads, we then used them to infer the consensus CDS sequence of each orthologous gene from $m-C D S$ in each species. The inferred nucleotide sequence at each site was estimated based on the number of mapped reads and the frequency of occurrence of each nucleotide at each site (Fig. 1). All sites were classified into one of 3 discrete categories (see Methods): 1) consensus sites (CS), encoded in [A, T, G, C]; 2) ambiguous sites (AS), encoded in $[\mathrm{a}, \mathrm{t}, \mathrm{g}, \mathrm{c}]$ and 3 ) uncovered site (UNS), encoded in gap character "-". We then estimated the consistency of CS sites across independent leaf RNA-Seq datasets from the same species. Among six species with both juvenile and mature leaf RNA-Seq datasets from 1KP Consortium, the highest consistency was found in F. bidentis, with entire predicted CS sites from mature leaf samples being identical to the corresponding CS sites from juvenile leaf. $99.99 \%$ of CS sites from $F$. trinervia were identical between juvenile and mature leaf. $F$. angustifolia, which had the lowest consistency, still showed $99.94 \%$ of predicted CS sites being equivalent between two types of leaf libraries (Additional file 1). Finally, the CS data matrix was built by concatenating CS sites that were shared in all samples (see Methods).

\section{The CS based phylogenetic inference method recapitulates previously published phylogenies}

Next, we examined whether CS based phylogenetic inference method can recapitulate previously published phylogenies. Here, we applied our method to the publicly available RNA-Seq data of 10 mosquito species from the genus Aedes and the genus Anopheles in Hittinger et al. [33]. The reference CDS of Aedes aegypti were used as mapping template. This dataset was selected because the divergence time between the genus Aedes and the genus Anopheles is $~ 108.4$ mya [36], which is comparable to the evolutionary time between $A$. thaliana and Flaveria. We obtained a data matrix comprising 251,184 CS spanning 1,678 genes in each species (see Methods). Both Maximum likelihood (ML) and Bayesian-inference (BI) were used to infer the phylogeny of the 10 mosquito species and the result showed that the bipartitions of the resulting $\mathrm{ML}$ and $\mathrm{BI}$ phylogenies were identical and both were the same as the published tree (Fig. 2).

\section{Constructed tree of individual samples of 17 Flaveria species based on $\mathrm{m}$-CDS}

Having demonstrated that the CS based phylogenetic inference method can recapitulate a published phylogeny in mosquito species with a well-described phylogeny, we then applied our method to RNA-Seq data from 34 individual samples of 17 Flaveria species. We obtained a CS data matrix comprising 315,342 CS sites from 2,183 genes (see Methods). BI tree and ML tree based on the CS data matrix had the same topology and showed the basal branching species were $\mathrm{C}_{3}$ and later branching species comprise the intermediates and $\mathrm{C}_{4}$ species (Additional file 2), which was consistent with the tree in [18]. In addition, there were also two obvious clades in our tree, only one of which contained the $\mathrm{C}_{4}$. However, compared with the tree in [18], our new tree showed a 

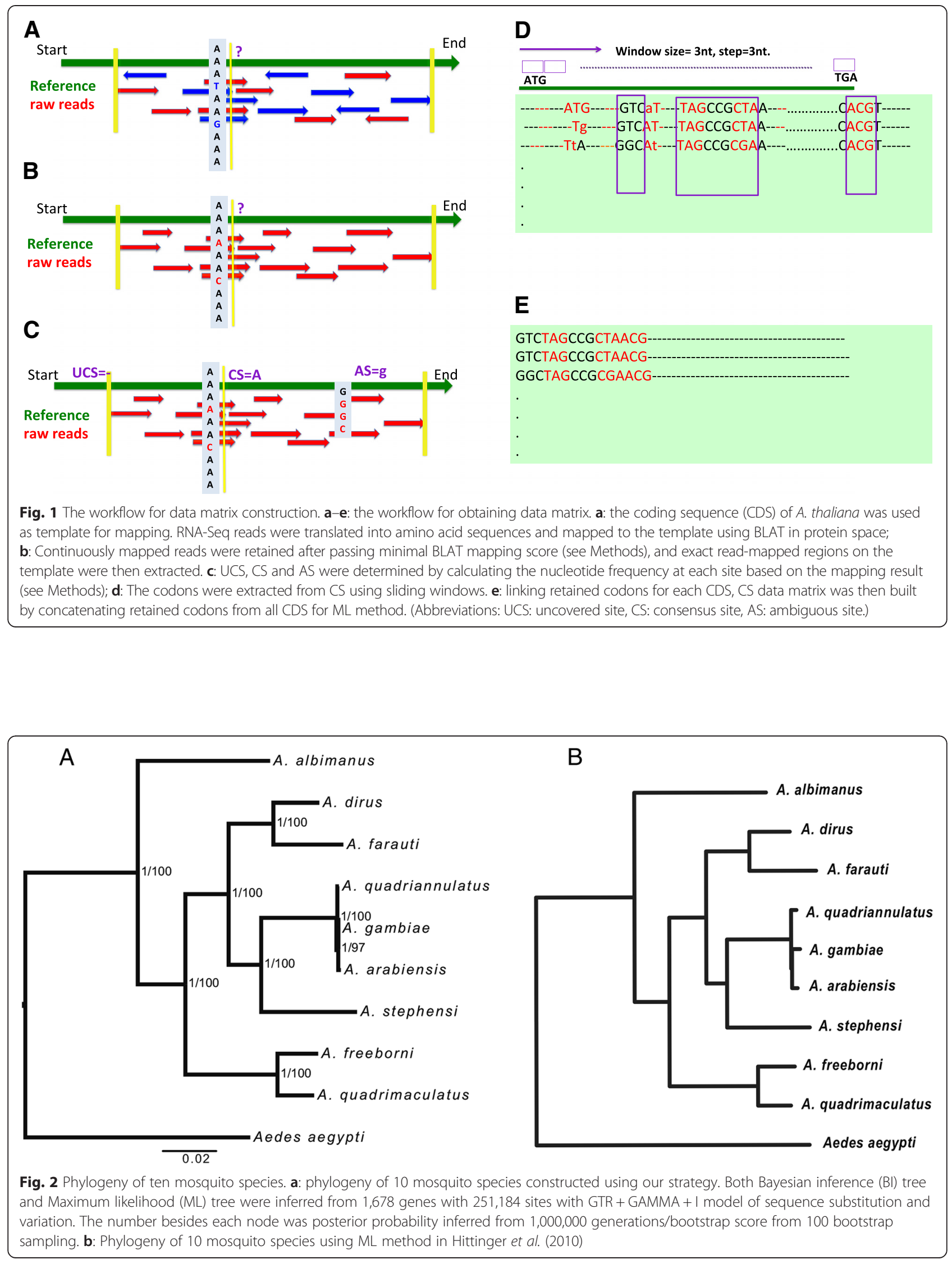
large shift in the position of $F$. angustifolia. The tree in [18] supported that $F$. angustifolia derived after the emergence of two clades which was supported by bootstrap score of 65 and Bayesian posterior probability of 65 . However, in our tree, it is predicted to have diverged before the appearance of the two distinct clades which was supported by bootstrap score of 100 and Bayesian posterior probability of 100 .

\section{The F. pringlei used in this study is likely a hybrid} between $\mathrm{F}$. pringlei and $\mathrm{F}$. angustifolia

It has previously been reported that F. pringlei $\left(\mathrm{C}_{3}\right)$ and F. angustifolia $\left(\mathrm{C}_{3}-\mathrm{C}_{4}\right)$ can hybridize [18]. In consistent with this, they branch as sister taxa in our tree, and importantly, F. pringlei from both HHU and 1KP Consortium were sister taxa in the tree based on individual Flaveria samples (Additional file 2). Therefore, it is possible that both samples of $F$. pringlei grown by HHU and University of Toronto teams may be from hybrid seeds of true $F$. pringlei and $F$. angustifolia. To assess this possibility, we defined $8126 C_{3}$ and $C_{3}-C_{4}$ marker sites from 3018 genes based on the pooled leaf samples (see Methods). Out of these 8126 sites, F. pringlei showed $9.04 \%$ being $C_{3}$ marker and $20.20 \%$ being $\mathrm{C}_{3}-\mathrm{C}_{4}$ marker, and $38 \%$ being hybrid site dominant by either $\mathrm{C}_{3}$ marker $(19.06 \%)$ or $\mathrm{C}_{3^{-}}$ $\mathrm{C}_{4}$ marker (19.00\%) (Table 2), indicating F. pringlei expressed genes both from $\mathrm{C}_{3}$ and $\mathrm{C}_{3}-\mathrm{C}_{4}$ species. In contrast, in F. angustifolia, $85.17 \%$ of sites were $\mathrm{C}_{3}-\mathrm{C}_{4}$ marker, which suggested that $F$. angustifolia belong to the $\mathrm{C}_{3}-\mathrm{C}_{4}$ species (Table 2). Similarly, by only analyzing $F$. pringlei sample from $\mathrm{HHU}$, we found $70 \%$ of marker sites have mapped reads being $\mathrm{C}_{3}$ and $\mathrm{C}_{3}-\mathrm{C}_{4}$ marker sites with a ratio close to be 1:1 (Additional file 3). Thus, our result suggested a high possibility of $F$. pringlei used by HHU was derived from a hybrid of true F. pringlei and F. angustifolia. Therefore, we termed $F$. pringlei in this study as $F$. pri $\times$ $F$. ang. To eliminate any potential influence on phylogeny construction caused by $F$. pri $\times F$. ang, we reconstructed phylogenetic tree without the $F$. pri $\times F$. ang samples.

\section{Constructed tree of 16 Flaveria species based on $\mathrm{m}$-CDS}

Based on the 31 RNA-Seq samples from 16 Flaveria species, we obtained a CS matrix comprising 343,590 CS sites from 2,190 genes (see Methods). Both the BI method and ML method were applied to infer the phylogeny based on CS matrix and the two methods generated exactly the same tree topology (Fig. 3). The tree excluding $F$. pri $\times F$. ang was largely congruent with the tree including F. pri $\times$ F. ang (Additional file 2) but presented two alternative branching possibilities: the taxon of $F$. sonorensis $\left(\mathrm{C}_{3}-\mathrm{C}_{4}\right)$ was exchanged with the taxon of $F$. angustifolia $\left(\mathrm{C}_{3}-\mathrm{C}_{4}\right)$, and the taxon of $F$. robusta $\left(\mathrm{C}_{3}\right)$ was exchanged with the taxon of $F$. cronquistii $\left(\mathrm{C}_{3}\right)$ (Fig. 3). The resulting topology was consistent with the topology in Mckown's tree [18], where F. cronquistii was the basal-branching taxon in Flaveria, and F. sonorensis emerged earlier than F. angustifolia. Therefore, we suggested a "dragging-to-root" effect on the position of $F$. angustifolia was caused by F. pri $\times F$. ang in the tree containing F. pri $\times F$. ang (Additional file 2). Notably, individual samples from different libraries of a species, such as samples from different tissues, different development stages, and different labs, were placed as the closet sister taxa (Fig. 3), suggesting that our method can be applied for RNA-Seq of plant samples from different sources or different sequencing protocols.

We found that some of the branches in the tree of Fig. 3 had low ML scores, e.g. the branch containing $F$. brownii and F. floridana $(\mathrm{ML}=48)$, and the branch containing $F$. bidentis, $F$. trinervia and $F$. australasica $(\mathrm{ML}=48)$. Given that trees inferred from the CS data matrix are independent of developmental stages, tissues, RNA-sequencing protocols, and growth conditions, we pooled all CS sites for the same species to increase the quantity of input data for phylogenetic tree construction. This pooling can potentially help resolve phylogenetic relationships for taxa of recent emergence. The pooled dataset comprised 20 species, including 16 Flaveria species and 3 out-group species together with $A$. thaliana, and the corresponding CS data matrix comprised 539,391 CS sites from 2,462 genes. As before, both ML and BI methods were used to infer the phylogeny using the CS data matrix (see Methods). The two approaches yielded identical tree topologies (Fig. 4). Importantly, the tree based on pooled samples (Fig. 4) was the same as the tree based on individual samples (Fig. 3). However, the bootstrap scores of this pooled-sample tree were on average higher than that of the tree based on individual samples,

Table 2 The percentage of sites with a $C_{3}$ origin, or $C_{3}-C_{4}$ origin in $F$. pri $\times F$. ang, $F$. angustifolia and $F$. sonorensis

\begin{tabular}{|c|c|c|c|c|c|c|}
\hline \multirow[b]{2}{*}{ Category } & \multicolumn{2}{|c|}{ Pulling F. pri $\times F$. ang $^{a}$} & \multicolumn{2}{|c|}{ F. angustifolia $\left(C_{3}-C_{4}\right)$} & \multicolumn{2}{|c|}{ F. sonorensis $\left(C_{3}-C_{4}\right)$} \\
\hline & \# sites & Proportion (\%) & \# sites & Proportion & \# sites & Proportion (\%) \\
\hline Expressed from $C_{3}$ allele & 731 & 9.04 & 764 & 9.76 & 0 & 0 \\
\hline Expressed from $C_{3}-C_{4}$ allele & 1633 & 20.2 & 6668 & 85.17 & 6609 & 97.62 \\
\hline Expressed from both alleles & 3075 & 38.03 & 18 & 0.23 & 0 & 0 \\
\hline Uncertain & 2573 & 31.83 & 305 & 4.84 & 161 & 3.38 \\
\hline
\end{tabular}

pulled RNA-Seq date sets from $\mathrm{HHU}$ and $1 \mathrm{KP}$ to interpret the Pulling F. pri $\times$ F. ang 


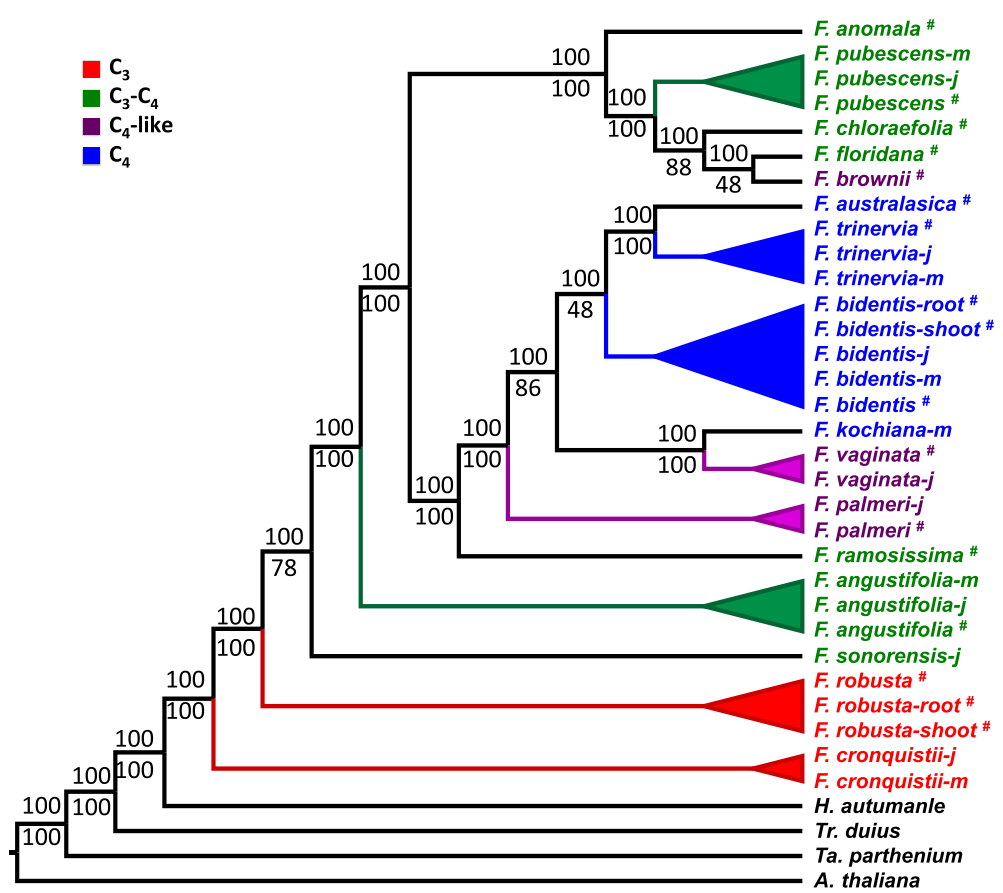

Fig. 3 Phylogenetic tree of individual Flaveria samples based on $m$-CDS. To remove the effect of $F$. pri $\times F$. ang on phylogenetic relationships among other species, the phylogenetic tree was constructed without F. pri $\times F$. ang. The $m$-CDS of $A$. thaliana was used as mapping reference to construct consensus sequence (CS) matrix according to Fig. 1. A CS matrix with 343,590 sites from 2,190 genes was used to infer phylogenetic relationships based on both Bayesian inference (BI) and Maximum likelihood (ML) using GTR + GAMMA + I model of sequence substitution and variation. BI tree and ML tree showed consistent topology. The numbers besides each node were posterior probability inferred from 1000,000 generations (up) and bootstrap score (down) from 500 bootstrap sampling. (\#/shoot\#/root\#/: leaf/shoot/root sample from HHU, j/m: juvenile/ mature leaf sample from 1 KP. m-CDS: reference contains the longest gene for each paralog family)

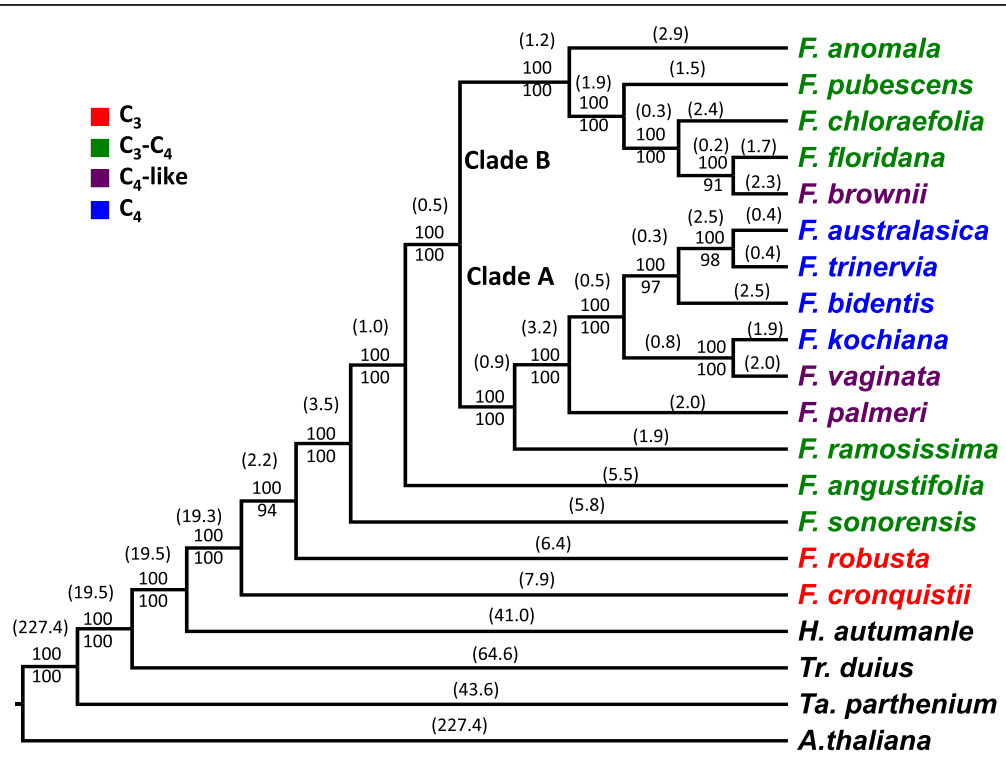

Fig. 4 Phylogenetic tree of 16 Flaveria species using $m$-CDS. Pooled RNA-Seq reads of 16 Flaveria species were mapped to $m$-CDS of $A$. thaliana, consensus sequence matric was then built according to method shown in Fig. 1. Both Bayesian inference (BI) tree and Maximum likelihood (ML) tree were inferred from 2, 462 genes with 539,391 sites with GTR + GAMMA + I model of sequence substitution and variation. The numbers besides each node were posterior probability (up) inferred from 1000,000 generations and bootstrap score (down) from 500 bootstrap sampling. The numbers in brackets were relative branch length estimated from Bayesian. ( $m$-CDS: reference contains the longest gene for each paralog family) 
especially at the nodes that were supported by low bootstrap scores. For example, the branch containing F. floridana and F. brownii was supported by bootstrap score of 48 in the individual-sample tree, but supported by a 91 bootstrap value in the pooled-sample tree.

It has been demonstrated previously that evolutionary rates may differ between single-copy and duplicated genes [37]. To determine whether this influenced our phylogeny, we created the singleton reference CDS $(s-C D S)$ dataset by removing all genes that have duplicates in A. thaliana from the $m$-CDS dataset (see Methods). The CS data matrix based on $s$-CDS containing 311,901 CS from 1,706 genes was then used to infer a phylogenetic tree of 16 Flaveria species by applying the same methods mentioned above (see Methods). The results showed that, the topology of the phylogenetic tree of 16 Flaveria species based on $s$-CDS (Additional file 4) was identical to that based on $m$-CDS (Fig. 4). But the tree based on $s$-CDS was supported by lower bootstrap scores, especially for those nodes that were supported by low bootstrap score in $m$-CDS. Our results indicate that multiple paralogs do not have a major effect on phylogeny outcomes based on RNA-Seq data.

It has been repeatedly demonstrated that $3^{\text {rd }}$ codon positions have different rates of evolution from those of the $1^{\text {st }}$ and $2^{\text {nd }}$ codon positions $[38,39]$. To estimate the effect of different codon position on the phylogenetic tree, we constructed separate phylogenetic trees using three codon positions independently (see Methods). Results showed that the trees based on the three independent codon positions and the entire codons were largely congruent with each other in most taxa; the major difference was observed in the positions of $F$. bidentis and $F$. angustifolia. Specifically, compared with trees based on the 2nd (Additional file $5 \mathrm{~B}$ ) and $3^{\text {rd }}$ codon positions (Additional file $5 \mathrm{C}$ ) as well as the entire codons (Fig. 4), the tree based on the $1^{\text {st }}$ codon position (Additional file $5 \mathrm{~A}$ ) placed $F$. bidentis at the base of $F$. kochiana and $F$. vaginata but with a low bootstrap score of 52 . The tree based on the $2^{\text {nd }}$ codon position, however, placed $F$. angustifolia at the base of clade A with a low bootstrap score of 74. Importantly, the topology of the phylogenetic tree inferred from the $3^{\text {rd }}$ codon position (Additional file $5 \mathrm{C}$ ) was identical to that based on entire codons (Fig. 4). This indicated that for the genus Flaveria, the strongest phylogenetic signal is derived from the 3rd codon position. We postulate that the short divergent time among Flaveria species ( $\sim$ mys) make the third codon position, which is most variable, a preferred choice in resolving the phylogenetic relationships according to [40]. This finding is consistent with previous reports that the 3rd position of codons contains the stronger phylogenetic signal $[38,39]$. In this regard, it is also worth mentioning that some reports [41, 42] suggested that the $3^{\text {rd }}$ position of codons can also potentially bias the construction of phylogenetic trees.

\section{Discussion}

\section{A new approach to construct phylogenetic trees using RNA-Seq data}

Phylogenetic analyses are essential for interpreting species relationships and evolutionary transitions within lineages, in particular, the origin of complex traits such as $\mathrm{C}_{4}$ photosynthesis. The advances of the next generation sequencing technologies are rapidly decreasing the cost of both transcritpome and whole genome sequencing. In this study, we exploited a novel method to use transcriptome data for phylogenetic inference. Compared to genome sequencing, RNA-Seq is much cheaper and is enriched in the genetic sequences that commonly form the basis of phylogenetic analysis [43]. Moreover, RNA-Seq is biased towards highly expressed genes, which are likely to have housekeeping and energy metabolism functions [44] and thus be conserved across species [33]. Therefore, RNA-Seq has been proposed as rich data in constructing construct phylogenetic trees to study evolutionary questions for living species [33].

In prior studies that have used RNA-seq to infer phylogenetic trees, a common first step has been to assemble short reads to generate sequence contigs, and then to map these contigs onto reference transcripts [33-35]. We used an alternative approach where we directly mapped RNASeq reads to the reference coding sequences (CDS) of the model species Arabidopsis thaliana (A. thaliana). This new method has a number of advantages: (1) it directly uses RNA-Seq reads for multiple sequence alignments in a single step and thus is suitable for scaling to a large number of species, (2) it bypasses the process of assembling short reads into contigs, thus saving computational resources and time, (3) it avoids potential errors caused by assembly, for example, misassembly or gene fusion, and (4) it avoids generating multiple sequence alignments and the potential errors introduced by this step. Although UTR and intronic regions are very useful in studying evolution, our method excludes such information because they are less conserved across species and therefore have less chance to be retained in data matrix after mapping short reads to CDS. Our method enables us to detect whether a sample is from a plant formed by hybridization of two other species, which is hard to assess using morphological data or a limited number of gene loci. This is crucial because samples from hybrid species may substantially influence the accuracy of a phylogeny. One drawback of our method is that ORFs may not be the same in Flaveria and in A. thaliana. To overcome this issue, we discarded genes with less than $10 \%$ of the CDS region being covered by CS sites.

Though RNA-Seq based approaches are shown to be effective, for now, PCR-based and EST-based methods 
are still preferred initial methods for resolving phylogenetic relationships, due to their relative ease and cost. RNA-Seq methods can serve as valuable alternatives when the phylogenetic relationship cannot be solved using traditional methods due to inadequate informative sites or short divergence time between species, or when it is hard to design PCR probes for species with little genomic information. Furthermore, our CS based phylogenetic inference approach can use samples from different tissues, living conditions, developmental stages, and sequencing protocols (Fig. 3). One potential caveat is that alternative splicing specific to some tissues may result in a different CS data matrix. Our results indicate that phylogenetic relationships of 16 Flaveria species were equivalent between the tree based on samples from different tissues (Fig. 3) and the tree derived from samples from only leaves (Fig. 4). Therefore, the effect of alternative splicing has relatively minor impacts on the construction of the phylogenetic tree using our method.

Multiple paralogs are a potential source of error in using RNA-Seq data to generate phylogenetic tree during the process of aligning reads to a reference. In this study, we devised strategies to avoid ambiguous results caused by paralogs. Specifically, we used two different references for reads mapping. The first reference used the longest gene for each paralog family $(m-C D S)$ in $A$. thaliana, and a separate reference used genes that have no paralogs in A. thaliana ( $s-C D S)$. Using $s-C D S$ references effectively remove cross mapping problems although at a cost of reduced numbers of informative sites. The procedure of consensus sequence (CS) construction further eliminated the influence of cross mapping reads because we accepted one site being a CS only if this site exists in at least 10 reads, of which $80 \%$ contains the same nucleotide. As a result, reads from paralogs, especially those with large sequence differences from the ancestor genes, should not contribute to the CS. The equivalent topology between the phylogenetic tree based on $m$-CDS and that based on $s$-CDS indicates that multiple paralogs have no effect on the topology RNA-Seq based phylogenies in the Flaveria case.

\section{An updated phylogeny of the genus Flaveria}

The updated tree (Fig. 4) from this study is largely consistent with the previously published tree [18]. Our new tree also shows two distinct clades in Flaveria (termed clades A and B by McKown et al. 2005 [18]). Our tree also indicates $C_{3}$ species branch in a basal position to intermediate and $\mathrm{C}_{4}$ species, thus supporting hypotheses that $\mathrm{C}_{3}$ photosynthesis is the ancestral condition in Flaveria [18]. One difference between the RNS-Seq tree and the tree in [18] is the position of F. angustifolia. In the previous published tree [18], F. angustifolia is placed at the root of clade B with a bootstrap score of $65(\mathrm{ML}=65)$ and Bayesian posterior probability of $65(\mathrm{BI}=65)$. However, in our trees, $F$. angustifolia resides in the root position for both clade A and clade B $(M L=100, B I=100)$, and this modification is consistent with the same number of layers (8 layers) of leaf ground tissue observed in F. angustifolia and two $C_{3}$ species: F. cronquistii and F. robusta [12]. Another difference is the place of $F$. chloraefolia in clade B, which was placed near the root of clade $\mathrm{B}$ branching above $F$. anomala $(\mathrm{ML}=56, \mathrm{BI}<50)$ in [18], however, the branch of $F$. chloraefolia was exchanged with the branch of $F$. pubescens $(\mathrm{ML}=100, \mathrm{BI}=100)$ in our tree. It is interesting to note that many of the ML bootstrap scores in the currently accepted tree for clade B are less than 80 in [18]. In contrast, in the updated tree, nodes in clade B have more reliable ML scores, with the lowest ML score being 91 (Fig. 4). Our tree agrees with [18] in the placement of the $F$. ramosissima branch in clade A, however, the branches containing F. kochiana and F. vaginanta were exchanged with the branch containing $F$. australasica and F. trinervia.

The main reason for these differences between the RNA-seq tree (Fig. 4) and the McKpwon et al. tree [18] is likely due to different number of informative sites available for two methods. The informative sites used in [18] may be insufficient, which resulted in lower supports based on ML or BI at some nodes, especially for nodes in clade B. As a result, the phylogenetic positions of F. brownii, F. linearis (A-D) and F. pubescens, F. oppositifolia, F. floridana were not resolved because of low ML and BI supports.

\section{Implications for $C_{4}$ evolution and engineering efforts based on the updated Flaveria phylogenetic tree}

The phylogenetic tree of the 16 Flaveria species suggested several features of $\mathrm{C}_{4}$ evolution (Fig. 4). First, clade A of the tree strongly supports hypotheses of stepwise evolution from $C_{3}$ towards $C_{4}$, which has been proposed based on morphological traits and physiological traits $[16,26,45]$. The progress from $C_{3}$ to $C_{4}$ photosynthesis in this clade started with a $\mathrm{C}_{3}-\mathrm{C}_{4}$ intermediate photosynthesis type in $F$. ramosissima and then to a $\mathrm{C}_{4}$-like photosynthesis type in F. palmeri [18]. Secondly, we found two parallel $\mathrm{C}_{4}$ sub-clades: the subclade containing $F$. kochiana and $F$. vaginata and the sub-clade containing $F$. bidentis, F. trinervia, and F. australasica. This repositioning presented two equally possible scenarios 1 ) that there was a single origin of $C_{4}$ photosynthesis with a reacquisition of weak $C_{3}$ activity in mesophyll cells in $F$. vaginata [16], or 2) that $C_{4}$ has arisen twice within this clade. Both hypotheses invoked two transitions and thus are equally likely [18]. Thirdly, clade $\mathrm{B}$ contained only intermediate species, i.e. $5 \mathrm{C}_{3}-\mathrm{C}_{4}$ species and one $\mathrm{C}_{4}$-like species, $F$. brownii, which shows comparable $\mathrm{C}_{4}$-like leaf anatomy and physiological traits 
as the F. palmeri $[18,46]$ in the clade A, suggesting that $\mathrm{C}_{4}$ traits can be recruited from multiple trajectories [47]. Such multiple trajectories towards $\mathrm{C}_{4}$ photosynthesis have also been previously proposed from phenotypic landscape inference $[10,19]$.

\section{Cross fertilization in the genus Flaveria}

Our results suggested that the $F$. pringlei (termed as F. pri $\times$ F. ang in this study) from both HHU and University of Toronto are hybrids from pure parents of a $F$. pringlei and a F. angustifolia (Table 2, Additional file 2). This could have happened either naturally in the field, or in cultivation in a greenhouse environment [25]. In addition to $F$. pringlei and F. angustifolia, many other Flaveria species are able to cross-fertilize, as have been summarized in Powell [25]. Therefore, it is important to check whether Flaveria samples are indeed pure species during experiments. Considering that none of the Flaveria genomes has been sequenced so far, comparing RNA-Seq reads with marker sites of defined photosynthetic types coupled with constructing a phylogeny using CS based phylogenetic inference approach provide a strategy to distinguish the hybridized or mixed samples from pure samples.

\section{Conclusions}

In this study, we developed a new procedure to obtain abundant phylogenetic data for generating phylogenetic trees in non-model species. Using this approach, we constructed a robust phylogeny of 16 Flaveria species, which were largely congruent with previous public trees although the positions of some species were modified. These modifications are supported with high branchsupports. We showed that samples of $F$. pringlei (termed as $F$. pri $\times F$. ang) used in a number of labs are hybrids of original $F$. pringlei $\left(\mathrm{C}_{3}\right)$ and $F$. angustifolia $\left(\mathrm{C}_{3}-\mathrm{C}_{4}\right)$. We propose that the new strategy on obtaining phylogenetic informative sequence from this study can be used to study phylogeny for a larger number of taxa.

\section{Methods}

\section{Sample preparation and high throughput sequencing}

Flaveria samples used in this study were from two sources. 16 leaf samples of 11 Flaveria species were grown in a greenhouse at University of Toronto and sequenced from One Thousand Plants (1KP) Consortium (https://sites.google.com/a/ualberta.ca/onekp/). Growth condition and plant treatment were described in detail in [48]. Leaves of two to four plants were sampled to obtain 0.1 to $1.0 \mathrm{~g}$ of tissue, with samples being flash frozen in liquid nitrogen and stored at $-80{ }^{\circ} \mathrm{C}$ until RNA extraction. For mature leaves, the newest fully expanded leaf was chosen. For juvenile leaves, the leaves most recently starting to expand from the main stem were chosen ( 0.1 to $0.5 \mathrm{~mm}$ depending on species). Samples were taken during June and July, during long daylengths of high light, and between 9 am and 1 pm. RNA was extracted using protocol 12 of [48]. Library construction was performed as an in-house service by BGI-Shenzhen using $20 \mu \mathrm{g}$ total RNA and the standard Illumina protocol. The second source of RNA-Seq samples was from 14. Flaveria species, including 14 leaf samples, two root samples and two shoot samples. Plant were grown in a greenhouse at Heinrich-Heine University (HHU) in 17$\mathrm{cm}$ pots of soil (C-400 with Cocopor [Stender Erden, Schermbeck Germany] and fertilized with 3 g/L Osmocote exact standard 3 to $4 \mathrm{M}$ [Scotts]). The plants received additional lighting to provide photoperiods of $16 \mathrm{~h}$ per day. The second and fourth visible leaves below the apex were harvested at noon and immediately frozen in liquid nitrogen and stored at $-80{ }^{\circ} \mathrm{C}$ until RNA extraction. Total RNA was isolated from the second and fourth leaves according to [49]. The remaining DNA was digested with DNAse for 15 min followed by phenol and chloroform extraction and precipitation. The RNA quality was tested on a DNA chip with the Agilent 2100 bioanalyzer. $1 \mu \mathrm{g}$ of total RNA was used for cDNA library generation with the TruSeq ${ }^{\mathrm{ma}}$ RNA Sample Preparation Kit (Illumina Lnc., San Diego, USA). Clusters were generated with the TruSeq SR Cluster Kit v2 according to the Reagent Preparation Guide with the Illumina cBot device. The RNA sequencing was performed with the Illumina platform. The RNA-Seq data of the 3 out-group species (Tragopogon duius, Helenium autumnale, and Tanacetum parthenium) were from $1 \mathrm{KP}$ Consortium. Their RNA isolation, library preparation and sequencing procedures were summarized in Johnson et al. [48], and the collection information is available at (https://sites.google.com/a/ualberta.ca/onekp/).

\section{Build minimal coding reference set and singleton coding reference set}

We used coding sequences (CDS) of Arabidopsis thaliana (A. thaliana) as reference for reads mapping because A. thaliana had the most comprehensive genome annotations for dicots. We used the CDS annotation from TAIR10 (http://www.arabidopsis.org/), which contained 35,368 references. To exclude potential artifacts from reads across-mapping between paralogs [30, 50], two reference CDS sets described below from A. thaliana were generated and used as templates for read mapping.

The first reference set we used was a minimal reference coding sequences $(m-C D S)$ set. In brief, the $m$-CDS set was built by cataloging paralogs into paralog groups and further retaining the longest gene for each paralog group. In order to remove all possible paralogs in the TAIR10 dataset (http://www.arabidopsis.org/), CDS from TAIR 10 were blasted in an "all against all" manner using the blastp program version 2.2.28 (blastall -p blastp) [51]. To choose 
a proper E-value cutoff for determining paralogs from blastp result, we exploited a set of known paralogs, which were predicted based on TAIR 9 dataset (http://www. arabidopsis.org/). The E-value of the upper $95 \%$ percentile of known paralogs was used as the cutoff to catalog possible paralogs into paralog groups from TAIR 10. Finally, only the longest gene from each paralog group was retained.

More stringently, considering genes with paralog (duplicates) may have different evolutionary rate compared with the genes without duplication (singleton) [37], a singleton reference coding sequences $(s-C D S)$ was generated as the second reference set, which was composed of singleton genes only.

\section{Read mapping and derived nucleotide sequence extrapolation}

RNA-Seq reads were mapped to $m$-CDS of $A$. thaliana using BLAT in protein space with the following parameters $[-\mathrm{t}=\mathrm{dnax}-\mathrm{q}=\mathrm{dnax}-$ repMatch $=100$-trimT] [52]. Then we defined the BLAT score cutoff for mapped reads based on the mapping false discovery rate (FDR). To estimate a FDR of mapping, we generated shuffled $m$-CDS by shuffling $m$-CDS by preserving hexamer frequency using Ushuffle $(k=6)$ [53]. FDR was estimated to be the ratio of the number of reads mapped to shuffled $m$-CDS to the number of reads mapped to $m-C D S$. Then at FDR less than $1 \%$, reads passed BLAT score cutoff (49 for 1KP samples and 39 for Tanacetum pathenium, and 54 from HHU samples) were considered as mapped reads.

Mapped reads were used to extrapolate the derived nucleotide sequence at each site for each gene in each sample (Fig. 1). Specifically, for each gene with aligned RNA-Seq reads, we calculated the frequency of each nucleotide [A, T, G, C] at each site. The derived nucleotide sequence at each site was extrapolated as follows: if a site had greater or equal to 10 mapped reads and no less than $80 \%$ of mapped reads reported the same nucleotide at this site e.g. "A", then the site was assigned to be a consensus site (CS) with confident nucleotide, i.e. "A". Otherwise, the site had no consensus nucleotide. The latter case can be further divided into two categories. If there was no read mapped to this site, the site was assigned to be an uncover site (UNS) and a symbol "-" was assigned to the site, and if there were reads mapped to this site, but either less than 10 reads mapped to or less than $80 \%$ of mapped reads reported the same nucleotide, the site was assigned as ambiguous site (AS) and lower letter of nucleotide reported by the nucleotide with the highest number, e.g. "a". Here we used $F$. bidentis as an example to illustrate this scenario. 10 reads were mapped to the second nucleotide site of the reference transcript AT5G54320.1 and eight of these reads reported
"A", therefore, the second site of this transcript was assigned as "A". Site 20 was assigned as "-" because no read was mapped to this site. The 30th site was assigned as "a" because 12 reads were mapped to this site, among them 8 reads mapped with "A" and 4 reads mapped with "T". Finally, only genes with CS sites covered at least $10 \%$ of their CDS lengths were retained in this study. To build CS data matrix, we first kept the CS sites only if they were CS sites across all samples, and then these CS were linked codon-by-codon for each CDS, which generated the CS data matrix. It should be noted that as all inferred CDS were constrained to the same $A$. thaliana reference sequence, therefore, there was no need for construction of multiple sequence alignments following concatenated sequence.

\section{Investigating consistency of CS of juvenile and mature leaf libraries from the same species}

To examine the accuracy of our CS extrapolation method, a proportion of identical CS sites between juvenile leaf and mature leaf of the same species were calculated for each of six species that their RNA-Seq samples were available for both developmental stages. For each transcript, the length of CS was first calculated from both juvenile leaf and mature leaf. The identical CS sites between the two libraries were then retained using in house Perl script, and the number of identical CS between juvenile leaf and mature leaf was plotted against the number of CS of mature leaves. The mean percentage was calculated for all transcripts in each species, shown at the bottom-right corner inside each panel figure (Additional file 1).

\section{Reconstructing phylogeny of ten mosquito species based on CS extrapolation method}

RNA-Seq data of ten mosquito species were retrieved from National Center for Biotechnology Information short read archive, with accession no. SRR031793 and SRR031794 for Anopheles stephensi, SRR031789 to SRR031792 for Anopheles quadrimaculatus, SRR031787 and SRR031788 for Anopheles freeborni, SRR031680 to SRR031682 for Anopheles arabiensis, SRR031706 and SRR031707 for Anopheles farauti, SRR031705 and SRR031691 for Anopheles dirus, SRR031667 and SRR031668 for Anopheles albimanus, SRR031659 to SRR031662 for Aedes aegypti, and SRR031663 to SRR031666 for Anopheles gambiae. Assembled cDNA reference of Aedes aegypti was downloaded from https://www.vectorbase.org/. CDS of Aedes aegypti was predicted based on assembled cDNA using Orfpredictor [54], which resulted in 18,469 predicted CDS from a total of $18,769 \mathrm{cDNA}$. RNA-Seq reads of ten mosquitoes were mapped to these 18,469 CDS reference of Aedes adgypti using BLAT with the parameters $[-\mathrm{t}=\mathrm{dnax}-\mathrm{q}=$ dnax - repMatch $=10000$-stepSize $=5$-minScore $=18$-trimT $]$ 
[52]. After constructing multiple sequence alignments using our method mentioned above, we then used both Maximum likelihood (ML) implemented in the RAxML package V. 2.2.3 [55] and Bayesian-inference (BI) from MrBayes 3.2.1 version [56] to infer the phylogeny of the ten mosquito species. In both cases, we used the GTR model of sequence evolution assuming a mutation rate following Gamma distribution with an estimate of proportion of invariant sites. Posterior probabilities in BI trees were calculated by running 1,000,000 generations and the first 2000 generations were used as burn-in. To construct the ML tree, genes were concatenated into a super gene in every species. The ML tree was inferred using the bootstrap parameter - $\mathrm{p} 1234$-b 1234 [55]. Bootstrap scores of ML tree were calculated by running 100 bootstrap samplings.

\section{CS-based phylogeny construction of 17 Flaveria species}

The CS-based phylogenetic data matrix was built using codons for which all their nucleotides were CS in all aligned samples. Data matrix based on individual RNASeq library of Flaveria species was constructed from $m$-CDS (Additional file 2). We used PartitionFinder (V1.1.1) [57] to estimate the proper model for inferring phylogeny based on obtained CS matrixes. The result showed GTR + GAMMA + I model (a General Time Reversible nucleotide substitution model with assumption that variations in sites follow gamma distribution and with a portion of invariant sites in a sequence) best fitted our datasets. This model was then used to infer phylogeny for each data matrix. Both ML and BI methods were used for phylogenetic tree construction. Posterior probabilities in BI trees were calculated by running 1000,000 generations and the first 2000 generations was used as burn-in. To construct ML tree, genes were concatenated into a super gene in every sample. The ML tree was inferred using bootstrap parameter: $-\mathrm{p} 1234$-b 1234 [55]. Bootstrap scores of ML trees were calculated by running 500 bootstrap samplings. The phylogenetic trees constructed using ML and BI methods were displayed using FigureTree (http://tree.bio.ed.ac.uk/software/ Figuretree/).

\section{Accessing hybrid results in Flaveria}

To investigate the possibility of the F. pringlei sample used in this study being a hybrid of true F. pringlei and F. angustifolia, we tested whether the $F$. pringlei sample in the present had 1:1 maternal and paternal alleles, in another word, we calculated the ratio between the number of sequences being $C_{3}$ maker sequence and the number of sequences being $\mathrm{C}_{3}-\mathrm{C}_{4}$ marker sequence in $F$. pringlei. Here, $C_{3}$ marker sequences were defined as the sequences that all $\mathrm{C}_{3}$ species were consistent (without considering F. pringlei), and $\mathrm{C}_{3}-\mathrm{C}_{4}$ marker sequences were defined as those sequences that all $\mathrm{C}_{3}-\mathrm{C}_{4}$ species were consistent (without considering $F$. angustifolia). For example, at site 872 of reference AT1G01710.1, $\mathrm{C}_{3}$ marker is $\mathrm{C}$, and $\mathrm{C}_{3}-\mathrm{C}_{4}$ marker is T. In F. pringlei, at this site, 58 \% of mapping reads showed $C$ and $42 \%$ of mapping reads showed $T$. Therefore, $F$. pringlei expressed genes showing both $\mathrm{C}_{3}$ and $\mathrm{C}_{3}-\mathrm{C}_{4}$ markers at this site. In this study, we classified sites as hybrid sites if they expressed both $\mathrm{C}_{3}$ and $\mathrm{C}_{3}-\mathrm{C}_{4}$ genes, with both being supported by no less than $40 \%$ of mapping reads. In contrast, if a site had no less than $90 \%$ of mapping reads reported be either a $\mathrm{C}_{3}$ or a $\mathrm{C}_{3}-\mathrm{C}_{4}$ marker, then this site was classified to be either a $C_{3}$ or a $\mathrm{C}_{3}-\mathrm{C}_{4}$ site. The third category of sites was that mapping reads showed neither $\mathrm{C}_{3}$ nor $\mathrm{C}_{3}-\mathrm{C}_{4}$ marker, which was then termed as a F. pringlei specific site. Based on this method, a large proportion of mixed sites observed in $F$. pringlei indicated a high probability that $F$. pringlei was from hybrid of pure $F$. pringlei and F. angustifolia. As a control, the number of sites belonging to each of these three categories in other two species, F. angusitoflia and F. sonorensis were calculated as well (Table 2).

\section{CS-based phylogeny construction of 16 Flaveria species}

CS data matrix of 16 Flaveria species (excluding F. pringlei) using individual RNA-Seq library based on $m$-CDS (Fig. 3) and using pooled RNA-Seq from different library based on $m$-CDS (Fig. 4) and $s$-CDS (Additional file 4) were constructed. Data matrix of 16 Flaveria species (excluding F. pringlei) based on $1^{\text {st }}, 2^{\text {nd }}$, and $3^{\text {rd }}$ codon positions were extracted from $m$-CDS using in-house Perl script (Additional file 5 A-C). We used PartitionFinder (V1.1.1) [57] to estimate the proper model for inferring phylogeny based on obtained CS data matrixes. The result showed GTR + GAMMA + I model best fitted these datasets. The same methods were then applied to infer the phylogeny based on these dataset respectively as mentioned above.

\section{Accession number}

The RNA-Seq datasets from 1KP Consortium were available at National Center for Biotechnology Information (NCBI) Gene Expression Omnibus (GEO) under accession number GSE54339. RNA-Seq data from HHU were available at NCBI Short Read Archive with accession number: SRX794138 for F. angustifolia, SRS777658 for F. floridana, SRS777663 for $F$. vaginata, SRS777671 for F. palmeri, SRS777680 for F. australasica, SRX468650 for F. robusta leaf, SRX794075 for F. robusta root, SRX794076 for $F$. robusta shoot, SRX468638 for F. pubescens, RX467630 for F. chloreafolia, SRX467620 for F. anomala, SRX467625 for F. brownii, SRX467614 for F. bidentis leaf, SRX794053 for F. bidentis root, SRX794064 for F. bidentis shoot, and SRX468662 for F. trinervia. 


\section{Additional files}

Additional file 1: The consistency of consensus sequence between mature leaf sample and juvenile leaf sample from the same species. The $X$-axis shows the number of consensus sequence (CS) for each gene in mature leaf sample (MS), Y-axis is the number of identical CS between MS and juvenile leaf sample (JS). At median, $99.96 \%$ sites were identical between MS and JS (Abbreviations: CS: consensus sequence, MS: mature leaf sample, JS: juvenile leaf sample).

Additional file 2: Phylogenetic tree based on individual Flaveria sample based on $m$-CDS. The $m$-CDS of $A$. thaliana that comprised only singleton genes was used as mapping reference to construct consensus sequence (CS) matrix according to Fig. 1. A CS matrix with 315,342 sites from 2,183 genes was used to infer phylogenetic relationships based on both Bayesian inference (BI) and Maximum likelihood (ML) using GTR + GAMMA + I model of sequence substitution and variation. BI tree and ML tree showed consistent topology. The numbers besides each node were posterior probability inferred from 1000,000 generations (up) and bootstrap score (down) from 500 bootstrap sampling (\#/shoot"/root": leaf/shoot/ root sample from HHU, $\mathrm{j} / \mathrm{m}$ : juvenile/mature leaf sample from $1 \mathrm{KP}$. $m$-CDS: reference contains the longest gene for each paralog family).

\section{Additional file 3: Estimation of the possibility of $F$. pringlei from}

HHU being a hybrid. A shows four types of sites in F. pringlei: 1) hybrid type, if $>=40 \%$ of mapping reads is same as the $C_{3}$ marker and $>=40 \%$ of mapping reads is same as $C_{3}-C_{4}$ marker. 2) $C_{3}-C_{4}$ type: if $>=90 \%$ of mapping reads is the same as $C_{3}-C_{4}$ marker. 3) $C_{3}$ type: if $>=90 \%$ of mapping reads is the same as the $C_{3}$ marker, and 4 ) others B shows the proportion of four types. F. pringlei had about $70 \%$ of sites being the hybrid type. $C$ shows the proportion of $\mathrm{C}_{3}$ marker and $\mathrm{C}_{3}-\mathrm{C}_{4}$ marker of each hybrid site in $F$. pringlei. The definition of $C_{3}$ marker and $\mathrm{C}_{3}-\mathrm{C}_{4}$ marker were defined in Methods.

\section{Additional file 4: Phylogeny of 16 Flaveria species pooling data} based on $s$-CDS. Pooling samples of the same species from different developmental stages resulted in samples representing 16 Flaveria species. The $s-C D S$ of $A$. thaliana was used as mapping reference to construct consensus sequence matrix. Both Bayesian inference (BI) tree and Maximum likelihood (ML) tree were inferred from 1,706 genes with 311,901 sites with GTR + GAMMA + I model of sequence substitution and variation. The numbers besides each node were posterior probability (up) inferred from 1000,000 generations and bootstrap score (down) from 500 bootstrap sampling. The numbers in brackets were relative branch length estimated from Bayesian ( $s$-CDS: reference contains only singleton genes).

\section{Additional file 5: Phylogenetic tree of 16 Flaveria species using} three independent codon positions from $m$-CDS. A: Phylogenetic tree based on $1^{\text {th }}$ codon sites. B: phylogenetic tree based on $2^{\text {nd }}$ codon sites. C: phylogenetic tree based on $3^{\text {rd }}$ codon sites. Pooling samples from different leaf libraries of one species resulted in samples representing 16 Flaveria species. Both Bayesian inference (BI) trees and Maximum likelihood (ML) tree were inferred from independent positions of codon from 2,271 genes with 191,482 sites using GTR+ GAMMA + I model of sequence substitution and variation. The numbers besides each node were posterior probability (up) inferred from 1000,000 generations and bootstrap score (down) from 500 bootstrap sampling ( $m$-CDS: reference contains the longest gene for each paralog family).

\section{Abbreviations}

mya: Million years ago; $m$-CDS: Minimal reference coding sequences; s-CDS: Singleton reference coding sequences; CS: Consensus sites; AS: Ambiguous sites; UNS: Uncovered site; M: Mature; J: Juvenile; ML: Maximum likelihood; BI: Bayesian inference.

\section{Competing interests}

The authors declare that they have no competing interests.

\section{Authors' contributions}

XGZ, RFS and PW designed the study, MJAL, UG, SK and MS carried out the computational analysis, RFS, SK, UG and PW grew the Flaveria species and prepared the RNA-Seq samples, SC and JM collected the Flaveria samples of
$1 \mathrm{KP}$ and $\mathrm{HHU}$ respectively, $\mathrm{HL}$, XW and GKSW performed Flaveria RNA-Seq sequencing from 1KP, MJAL and XGZ wrote the manuscript, UG, SK, PW, $J M H, R F S$ and GKSW revised the manuscript. All authors read and approved the final manuscript.

\section{Acknowledgement}

We give our thanks to Prof. Antonis Rokas for offering the RNA-Seq source of ten mosquitoes. We thank Hai Yang Hu, Yimin Tao, Yuanyuan Li and Jiajia Xu for helpful discussion. We also give thanks to anonymous reviewers for helpful suggestions and comments. GKSW acknowledges the support of Alberta Enterprise and Advanced Education, Genome Alberta, Alberta Innovates Technology Futures iCORE, Musea Ventures, and BGI-Shenzhen. Computations were enabled by facilities at WestGrid, through Compute/ Calcul Canada, and at Texas Advanced Computing Center, through iPlant Collaborative. SK is a Leverhulme Trust Early Career Fellow. This work was supported by Bill \& Melinda Gates Foundation [OPP1014417 to Xin-Guang Zhu]; National Science Foundation of China [30970213 to Xin-Guang Zhu]; Ministry of Science and Technology of China [2011DFA31070 to Xin-Guang Zhu]; and the Young Talent Frontier Program of Shanghai Institutes for Biology Sciences / Chinese Academy of Sciences [09Y1C11501 to Xin-Guang Zhu].

\section{Author details}

'CAS-MPG Partner Institute and Key Laboratory for Computational Biology, Shanghai Institutes for Biological Sciences, Shanghai, China. ${ }^{2}$ Institute of Plant Molecular and Developmental Biology, Heinrich-Heine-University, Dusseldorf, Germany. ${ }^{3}$ Department of Plant Sciences, University of Oxford, Oxford, UK. ${ }^{4}$ Department of Plant Sciences, University of Cambridge, Cambridge, UK ${ }^{5}$ Department of Ecology and Evolutionary Biology, University of Toronto, Toronto, Canada. ${ }^{6} \mathrm{BGI}$-Shenzhen, Beishan Industrial Zone, Yantian District, Shenzhen 518083, China. ${ }^{7}$ Department of Biological Sciences, University of Alberta, Edmonton, AB T6G 2E9, Canada. ${ }^{8}$ Department of Medicine, University of Alberta, Edmonton, AB T6G 2E1, Canada.

Received: 4 December 2014 Accepted: 29 May 2015

Published online: 18 June 2015

\section{References}

1. Sage RF, Sage $T L$, Kocacinar F. Photorespiration and the evolution of $C_{4}$ photosynthesis. Annu Rev Plant Biol. 2012;63:19-47.

2. Sage TL, Busch FA, Johnson DC, Friesen PC, Stinson CR, Stata M, et al. Initial events during the evolution of $C_{4}$ photosynthesis in $C_{3}$ species of Flaveria. Plant Physiol. 2013;163(3):1266-76.

3. Soros $\mathrm{CL}$, Dengler NG. Ontogenetic derivation and cell differentiation in photosynthetic tissues of $C_{3}$ and $C_{4}$ Cyperaceae. Am J Bot. 2001;88(6):992-1005.

4. Sage RF, Coleman JR. Effects of low atmospheric $\mathrm{CO}_{2}$ on plants: more than a thing of the past. Trends Plant Sci. 2001;6(1):18-24.

5. Kanai REG. The biochemistry of $C_{4}$ photosynthesis. In: Sage RF, Monson RK, editors. $C_{4}$ Plant Biology. San Diego: Academic Press; 1999. p. 49-87.

6. Sage RF, Zhu XG. Exploiting the engine of $C_{4}$ photosynthesis. J Exp Bot. 2011;62(9):2989-3000.

7. Sage RF. Environmental and evolutionary preconditions for the origin and diversification of the $C_{4}$ photosynthetic syndrome. Plant Biol. 2001;3:202-13.

8. Johnson $\mathrm{HS}$, Hatch MD. The $\mathrm{C}_{4}$-dicarboxylic acid pathway of photosynthesis, Identification of intermediates and products and quantitative evidence for the route of carbon flow. Biochem J. 1969;114(1):127-34.

9. Langdale JA. $C_{4}$ cycles: past, present, and future research on $C_{4}$ photosynthesis. Plant Cell. 2011;23(11):3879-92.

10. Hibberd JM, Sheehy JE, Langdale JA. Using $C_{4}$ photosynthesis to increase the yield of rice-rationale and feasibility. Curr Opin Plant Biol. 2008;11(2):228-31.

11. Sage RF. The evolution of $C_{4}$ photosynthesis. New Phytol. 2003;161:2

12. McKown AD, Dengler NG. Key innovations in the evolution of Kranz anatomy and $\mathrm{C}_{4}$ vein pattern in Flaveria (Asteraceae). Am J Bot. 2007;94(3):382-99.

13. Monson RK, Bd M, Ku MSB, Edwards GE. Co-function of $\mathrm{C}_{3}-$ and $C_{4}$-photosynthetic pathways in $C_{3}, C_{4}$ and $C_{3}-C_{4}$ intermediate Flaveria species. Planta. 1986;168:493-502.

14. Vogan PJ, Frohlich MW, Sage RF. The functional significance of $\mathrm{C}_{3}-\mathrm{C}_{4}$ intermediate traits in Heliotropium L. (Boraginaceae): gas exchange perspectives. Plant Cell Environ. 2007;30(10):1337-45. 
15. Gowik U, Brautigam A, Weber KL, Weber AP, Westhoff P. Evolution of $C_{4}$ photosynthesis in the genus Flaveria: how many and which genes does it take to make $C_{4}$ ? Plant Cell. 2011;23(6):2087-105.

16. Vogan PJ, Sage RF. Water-use efficiency and nitrogen-use efficiency of $C_{3}-C_{4}$ intermediate species of Flaveria Juss. (Asteraceae). Plant Cell Environ. 2011;34(9):1415-30.

17. Heckmann D, Schulze S, Denton A, Gowik U, Westhoff P, Weber AP, et al. Predicting $C_{4}$ photosynthesis evolution: modular, individually adaptive steps on a Mount Fuji fitness landscape. Cell. 2013;153(7):1579-88.

18. McKown AD, Moncalvo JM, Dengler NG. Phylogeny of Flaveria (Asteraceae) and inference of $C_{-4}$ photosynthesis evolution. Am J Bot. 2005;92(11):1911-28.

19. Williams BP, Johnston IG, Covshoff S, Hibberd JM. Phenotypic landscape inference reveals multiple evolutionary paths to $C_{4}$ photosynthesis. eLife. 2013;2:e00961.

20. Ku MS, Wu J, Dai Z, Scott RA, Chu C, Edwards GE. Photosynthetic and photorespiratory characteristics of flaveria species. Plant Physiol. 1991;96(2):518-28.

21. Brown RH, Bassett CL, Cameron RG, Evans PT, Bouton JH, Black CC, et al. Photosynthesis of $F_{1}$ hybrids between $C_{4}$ and $C_{3}-C_{4}$ species of Flaveria. Plant Physiol. 1986;82(1):211-7.

22. Gowik U, Burscheidt J, Akyildiz M, Schlue U, Koczor M, Streubel M, et al. cis-Regulatory elements for mesophyll-specific gene expression in the $C_{4}$ plant Flaveria trinervia, the promoter of the $C_{4}$ phosphoenolpyruvate carboxylase gene. Plant Cell. 2004;16(5):1077-90.

23. Brown $\mathrm{RH}$, Byrd GT, Black CC. Degree of $C_{4}$ photosynthesis in $C_{4}$ and $\mathrm{C}_{3}-\mathrm{C}_{4}$ Flaveria species and their hybrids: II. Inhibition of apparent photosynthesis by a phosphoenolpyruvate carboxylase inhibitor. Plant Physiol. 1992;100(2):947-50.

24. Schulze S, Mallmann J, Burscheidt J, Koczor M, Streubel M, Bauwe H, et al. Evolution of $\mathrm{C}_{4}$ photosynthesis in the genus Flaveria: establishment of a photorespiratory $\mathrm{CO}_{2}$ pump. Plant Cell. 2013;25(7):2522-35.

25. Powell AM. Systematics of Flaveria (Flaveriinae-Asteraceae). Ann Mo Bot Gard. 1978;65:590-636.

26. Kopriva S, Chu CC, Bauwe H. Molecular phylogeny of Flaveria as deduced from the analysis of nucleotide sequences encoding the $\mathrm{H}$-protein of the glycine cleavage system. Plant Cell Environ. 1996;19(9):1028-36.

27. Mason-Gamer RJ, Kellogg EA. Testing for phylogenetic conflict among molecular data sets in the tribe Triticeae (Gramineae). Syst Biol. 1996;45(4):524-45.

28. Kellogg EA, Appels R. Intraspecific and interspecific variation in 5S RNA genes are decoupled in diploid wheat relatives. Genetics. 1995;140(1):325-43.

29. Petkovits T, Nagy LG, Hoffmann K, Wagner L, Nyilasi I, Griebel T, et al. Data partitions, Bayesian analysis and phylogeny of the zygomycetous fungal family Mortierellaceae, inferred from nuclear ribosomal DNA sequences. PLoS One. 2011;6(11):e27507.

30. Brautigam A, Gowik U. What can next generation sequencing do for you? Next generation sequencing as a valuable tool in plant research. Plant Biol (Stuttg). 2010;12(6):831-41.

31. Neves SS, Forrest LL. Plant DNA sequencing for phylogenetic analyses: from plants to sequences. Methods Mol Biol. 2011;781:183-235.

32. Boussau B, Szollosi GJ, Duret L, Gouy M, Tannier E, Daubin V. Genome-scale coestimation of species and gene trees. Genome Res. 2012;23:323-30.

33. Hittinger CT, Johnston M, Tossberg JT, Rokas A. Leveraging skewed transcript abundance by RNA-Seq to increase the genomic depth of the tree of life. Proc Natl Acad Sci U S A. 2010;107(4):1476-81.

34. Johnson KP, Walden KK, Robertson HM. Next-generation phylogenomics using a target restricted assembly method. Mol Phylogenet Evol. 2013;66(1):417-22.

35. Zou M, Guo B, Tao W, Arratia G, He S. Integrating multi-origin expression data improves the resolution of deep phylogeny of ray-finned fish (Actinopterygii). Sci Rep. 2012;2:665.

36. Hedges SB, Dudley J, Kumar S. TimeTree: a public knowledge-base of divergence times among organisms. Bioinformatics. 2006;22(23):2971-2.

37. Jordan IK, Wolf YI, Koonin EV. Duplicated genes evolve slower than singletons despite the initial rate increase. BMC Evol Biol. 2004;4:22.

38. Simmons MP, Zhang LB, Webb CT, Reeves A. How can third codon positions outperform first and second codon positions in phylogenetic inference? An empirical example from the seed plants. Syst Biol. 2006;55(2):245-58

39. Christianson ML. Codon usage patterns distort phylogenies from or of DNA sequences. Am J Bot. 2005;92(8):1221-33.
40. Hillis DM. Molecular versus morphological approaches to systematics. Annu Rev Ecol Syst. 1987;18:23-42.

41. Breinholt JW, Kawahara AY. Phylotranscriptomics: saturated third codon positions radically influence the estimation of trees based on next-gen data. Genome Biol Evol. 2013;5(11):2082-92.

42. $X_{i}$ Z, Liu L, Rest JS, Davis CC. Coalescent versus concatenation methods and the placement of Amborella as sister to water lilies. Syst Biol. 2014;63(6):919-32.

43. Zhang G, Liu X, Quan Z, Cheng S, Xu X, Pan S, et al. Genome sequence of foxtail millet (Setaria italica) provides insights into grass evolution and biofuel potential. Nat Biotechnol. 2012;30(6):549-54.

44. Gibbons JG, Janson $E M$, Hittinger $C T$, Johnston $M$, Abbot $P$, Rokas $A$. Benchmarking next-generation transcriptome sequencing for functional and evolutionary genomics. Mol Biol Evol. 2009;26(12):2731-44.

45. Sudderth EA, Muhaidat RM, McKown AD, Kocacinar F, Sage RF. Leaf anatomy, gas exchange and photosynthetic enzyme activity in Flaveria kochiana. Funct Plant Biol. 2007;34(2):118-29.

46. Cheng SH, Moore BD, Edwards GE, Ku MS. Photosynthesis in Flaveria brownii, a $\mathrm{C}_{4}$-like species: leaf anatomy, characteristics of $\mathrm{CO}_{2}$ exchange, compartmentation of photosynthetic enzymes, and metabolism of $\mathrm{CO}_{2}$. Plant Physiol. 1988;87(4):867-73.

47. Beer KD, Orellana MV, Baliga NS. Modeling the evolution of $C_{4}$ photosynthesis. Cell. 2013;153(7):1427-9.

48. Johnson MT, Carpenter EJ, Tian Z, Bruskiewich R, Burris JN, Carrigan CT, et al. Evaluating methods for isolating total RNA and predicting the success of sequencing phylogenetically diverse plant transcriptomes. PLoS One. 2012;7(11):e50226.

49. Westhoff P, Offermann-Steinhard K, Hofer M, Eskins K, Oswald A, Streubel M. Differential accumulation of plastid transcripts encoding photosystem ॥ components in the mesophyll and bundle-sheath cells of monocotyledonous NADP-malic enzyme-type C 4 plants. Planta. 1991;184(3):377-88.

50. Brautigam A, Kajala K, Wullenweber J, Sommer M, Gagneul D, Weber KL, et al. An mRNA blueprint for $C_{4}$ photosynthesis derived from comparative transcriptomics of closely related $C_{3}$ and $C_{4}$ species. Plant Physiol. 2011;155(1):142-56.

51. Altschul SF, Madden TL, Schaffer AA, Zhang J, Zhang Z, Miller W, et al. Gapped BLAST and PSI-BLAST: a new generation of protein database search programs. Nucleic Acids Res. 1997;25(17):3389-402.

52. Kent WJ. BLAT-the BLAST-like alignment tool. Genome Res. 2002;12(4):656-64.

53. Jiang M, Anderson J, Gillespie J, Mayne M. uShuffle: a useful tool for shuffling biological sequences while preserving the k-let counts. BMC Bioinf. 2008;9:192.

54. Min XJ, Butler G, Storms R, Tsang A. OrfPredictor: predicting protein-coding regions in EST-derived sequences. Nucleic Acids Res. 2005;33(Web Server issue):W677-80

55. Stamatakis A. RAxML-VI-HPC: maximum likelihood-based phylogenetic analyses with thousands of taxa and mixed models. Bioinformatics. 2006;22(21):2688-90.

56. Ronquist F, Teslenko M, van der Mark P, Ayres DL, Darling A, Hohna S, et al. MrBayes 3.2: efficient Bayesian phylogenetic inference and model choice across a large model space. Syst Biol. 2012;61(3):539-42.

57. Lanfear R, Calcott B, Ho SY, Guindon S. Partitionfinder: combined selection of partitioning schemes and substitution models for phylogenetic analyses. Mol Biol Evol. 2012;29(6):1695-701.

\section{Submit your next manuscript to BioMed Central and take full advantage of:}

- Convenient online submission

- Thorough peer review

- No space constraints or color figure charges

- Immediate publication on acceptance

- Inclusion in PubMed, CAS, Scopus and Google Scholar

- Research which is freely available for redistribution 